\title{
MicroRNA-497 inhibits multiple myeloma growth and increases susceptibility to bortezomib by targeting Bcl-2
}

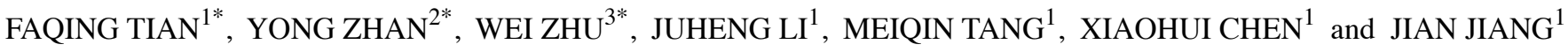 \\ ${ }^{1}$ Department of Hematology, Longgang District People's Hospital of Shenzhen, Shenzhen, Guangdong 518172; \\ ${ }^{2}$ Department of Pathology, School of Basic Medicine, Guangdong Medical University, Dongguan, Guangdong 523808; \\ ${ }^{3}$ Department of Radiology, Longgang District People's Hospital of Shenzhen, Shenzhen, Guangdong 518172, P.R. China
}

Received February 2, 2018; Accepted October 30, 2018

DOI: $10.3892 /$ ijmm.2018.4019

\begin{abstract}
Multiple myeloma (MM) is a common severe hematopoietic malignancy occuring in aged population. MicroRNA (miR)-497 was previously reported to contribute to the apoptosis of other cell types, presumably through targeting B-cell lymphoma 2 (Bcl-2). In the present study, miRNA and protein expression levels were detected by reverse transcription-quantitative polymerase chain reaction and western blot analyses, respectively. The cell proliferation and viability was measured using 3-(4,5-dimethylthiazol-2-yl)-2, 5-diphenyltetrazolium bromide and plate clonality assays, and the cell growth cycle was measured with a flow cytometer. Terminal deoxynucleotidyl transferase (TdT)-mediated dUTP nick-end-labeling, Annexin V and caspase-3 activity assays were performed to examine the cell apoptotic rates. The results showed that miR-497 was markedly decreased, whereas Bcl-2 was enhanced in MM tissues and cell lines. miR-497 targeted Bcl-2 and affected its downstream apoptosis-related genes. The overexpression of miR-497 promoted MM cell apoptosis through cell cycle arrest, and decreased colony genesis ability and viability. In addition, miR-497 increased the sensitivity of MM cells to bortezomib. Taken together, miR-497 suppressed MM cell proliferation and promoted apoptosis by directly targeting Bcl-2 and altering the expression of downstream apoptosis-related proteins. The combination of miR-497 and bortezomib may enhance drug sensitivity, serving as a potentially available therapeutic method for MM.
\end{abstract}

Correspondence to: Dr Faqing Tian, Department of Hematology, Longgang District People's Hospital of Shenzhen, 53 Ai Xin Road, Longgang, Shenzhen, Guangdong 518172, P.R. China

E-mail: tianfaqing110@sina.com

*Contributed equally

Key words: microRNA-497, multiple myeloma, B-cell lymphoma 2, apoptosis, bortezomib

\section{Introduction}

Multiple myeloma (MM) is a common hematological malignancy caused by the malignant proliferation of plasma cells (PCs), which accumulate within bone marrow $(1,2)$. As a consequence, normal hematopoiesis is disrupted. MM ranks as the second most common of all hematological malignancies around the world (3). However, no specific markers have been validated for MM detection or therapy, representing an obstacle for the investigation of MM. Although several studies in previous years have revealed certain therapeutics, such progress remains inadequate, and investigations of MM are urgently required.

MicroRNAs (miRNAs) are small, non-coding RNAs with a length of $\sim 22$ nucleotides. They are considered to function as mRNA regulators by interacting with the 3 '-untranslated region (3'UTR) of the targeted mRNA transcript and leading to degradation (2-5). Several, if not all, biological processes are reported to be regulated by miRNAs, including cell cycle, proliferation, metabolism, immune response, hematopoiesis and differentiation (6-10). It is widely accepted that miRNAs are vital in the process of tumorigenesis by targeting key factors $(11,12)$. They are also considered as potential therapeutic factors for several diseases.

miR-497 is one of the well-studied miRNAs and is considered to be a tumor-suppressive miRNA in osteosarcoma, colorectal carcinoma, glioma, breast carcinoma and several other cancer types, being involving in the regulation of tumor cell growth, migration, invasion and chemoresistance (11-17). Previous studies have found that miR-497 was downregulated in patients with MM compared with healthy donors $(18,19)$, and miR-497 was the one of four miRNA signatures identified as having differential expression levels that correlated with lenalidomide/dexzmethasone treatment response in a primary plasma cell leukemia clinical trial (19). However, the role and underlying mechanism of miR-497 in regulating the tumorigenesis of leukemia remain to be fully elucidated.

Multiple genes may be targets of miR-497, and a well-accepted potential target of miR-497 is B-cell lymphoma 2 (Bcl-2) $(15,20-23)$. Bcl-2 is a protein involved in modulating cell death through suppressing apoptosis and promoting cell survival. In several types of cancer, $\mathrm{Bcl}-2$ is reported to be 
upregulated $(23,24)$. Its expression is also considered to be regulated by multiple miRNAs, including miR-497. Bcl-2 family proteins are also involved in the chemoresistance of MM (9).

Few studies have reported the functional mechanism of miR-497 and Bcl-2 in MM, particularly whether miR-497 can directly target $\mathrm{Bcl}-2$ and influence $\mathrm{MM}$ cell cycle and apoptosis. In addition, as miR-497 and $\mathrm{Bcl}-2$ are involved in chemotherapy of other types of cancer $(20,21,23)$, it is of interest to evaluate the possibility of the combination therapy of miR-497 and bortezomib, a common drug used for MM treatment (25). Although bortezomib promotes the death of malignant plasma cells and is a conventional clinical medication used in MM therapy, several patients are intrinstically or become bortezomib-resistant $(26,27)$. Therefore, RPMI-8226 and U266 MM cell lines, which are confirmed to be resistant to bortezomib $(26,27)$, were used in the present study to verify the function of miR-497. The results may reveal a synergetic effect of miR-497 in improving bortezomib efficacy.

\section{Materials and methods}

Clinical specimens. Bone marrow aspirates of 30 untreated II phase MM patients (50-70 years old, half men and half women, serum $\beta$-microglobulin=3.5-5.5 mg/l) were collected at the Longgang District People's Hospital of Shenzhen (Shenzhen, China) from September 2016 to December 2017. Normal bone marrow aspirates were collected from 30 healthy donors with signed statements of informed consent. The bone marrow aspirates were lysed to obtain the bone marrow mononuclear cells (BMMCs), and then the total RNA were extracted by commercial kits for subsequent gene expression analysis. The ethics committee of the Longgang District People's Hospital of Shenzhen approved the present study.

Cell culture. The RPMI-8266 and U266 human MM cell lines were obtained from American Type Culture Collection (Manassas, VA, USA) and cultured in RPMI-1640 medium supplemented with $10 \%$ fetal bovine serum (FBS; Gibco; Thermo Fisher Scientific, Inc., Waltham, MA, USA) in a humidified atmosphere of $95 \%$ air and $5 \% \mathrm{CO}_{2}$ at $37^{\circ} \mathrm{C}$. The bone mesenchymal stem cells (BMSCs) were isolated from human bone segments, as described previously, and cultured in a standard growth medium for stem cells (28).

miRNA transfection and drug treatment. The human miR-497 mimic (miR-497) and the negative control (miR-NC) were designed and synthesized by GenePharma (Shanghai, China). When the cells were cultured to $80 \%$ confluence, the RNA oligonucleotides were transfected into the cells using Lipo3000 (Invitrogen; Thermo Fisher Scientific, Inc.) according to the manufacturer's protocol. The two miRNAs were used at $50 \mathrm{nM}$ unless otherwise indicated.

For the drug susceptibility assay, bortezomib was dissolved in DMSO at a storage concentration of $10 \mu \mathrm{M}$ and administered following miRNA transfection for $24 \mathrm{~h}$ with a concentration range of $0,5,10,15$, and $20 \mathrm{nM}$ when cells reached $90 \%$ confluency. Following another $24 \mathrm{~h}$ incubation with bortezomib or DMSO at $37^{\circ} \mathrm{C}$, the cells were collected for analysis.

3-(4,5-Dimethylthiazol-2-yl)-2,5-diphenyltetrazolium bromide (MTT) assay. An MTT assay was performed for cell viability detection. The cells were seeded into a 96 -well plate for culture with serum-free medium for $24 \mathrm{~h}$. Following treatment, the cells were rinsed with PBS and treated with $20 \mu \mathrm{l}$ of $5 \mathrm{mg} / \mathrm{ml}$ MTT for $4 \mathrm{~h}$ at $37^{\circ} \mathrm{C}$, and DMSO was used to dissolve the formazan precipitation. The absorbance at $570 \mathrm{~nm}$ was measured using a plate reader, and the cell viability was calculated as follows: Absorbance $(A)=\left(A_{\text {treatemnt }}-\mathrm{A}_{\text {control }}\right) / \mathrm{A}_{\text {control }} \times 100 \%$.

PI staining for cell cycle analysis. The cells were rinsed with PBS, trypsinized and collected by centrifugation at $500 \mathrm{x} \mathrm{g}$ for $5 \mathrm{~min}$ and room temperature $\left(25^{\circ} \mathrm{C}\right)$. Following discarding of the supernatant, the cells were resuspended with PBS and rinsed for 3 times, and then ice-cold ethanol was added to fix the cells at $-20^{\circ} \mathrm{C}$ overnight. Following centrifugation $\left(500 \times \mathrm{g}, 5 \mathrm{~min}, 25^{\circ} \mathrm{C}\right)$ and rinse of 3 times, $1 \times 10^{6}$ cells were added to a mix of $400 \mu \mathrm{l} \mathrm{RNase}(20 \mu \mathrm{g} / \mathrm{ml})$ together with PI $(50 \mu \mathrm{g} / \mathrm{ml})$ and $0.2 \%$ Triton X-100 in PBS, and then incubated for $30 \mathrm{~min}$ at $37^{\circ} \mathrm{C}$. Finally, the cells were collected, rinsed and centrifuged again $\left(500 \mathrm{x} \mathrm{g}, 5 \mathrm{~min}, 25^{\circ} \mathrm{C}\right)$, and then resuspended with PBS for flow cytometric analysis.

Apoptosis assay. Annexin V-FITC was used for the analysis of apoptosis assay following the manufacturer's protocol. The cells were rinsed with cold PBS three times. Following centrifugation at $500 \mathrm{x} \mathrm{g}$ for $5 \mathrm{~min}$ at $4^{\circ} \mathrm{C}$, the cells were collected and concentrated to $1 \times 10^{5}$ cells per $\mathrm{ml}$. The sample solution $(0.1 \mathrm{ml})$ was mixed with $5 \mu \mathrm{l}$ FITC-conjugated Annexin $\mathrm{V}$ and $5 \mu \mathrm{l}$ PI solution. The mixture was incubated for $30 \mathrm{~min}$ in the dark at room temperature, and then analyzed by flow cytometry.

Soft-agar colony formation assay. For the soft-agar colony formation assay, the cells were mixed with DMEM containing $0.35 \%$ low-melting agarose and $10 \%$ FBS, and seeded 500 cells onto a coating of $0.7 \%$ low-melting agarose in DMEM with $10 \%$ FBS. The cells were incubated for up to 3 weeks, following which colonies were visualized by $0.4 \%$ crystal violet and imaged using a digital camera (D5600; Nikon Corporation, Tokyo, Japan). Colonies with $>0.1 \mathrm{~mm}$ diameter were considered as positive.

Terminal deoxynucleotidyl transferase (TdT)-mediated dUTP nick-end-labeling (TUNEL) assay. A TUNEL assay was performed using an in situ Cell Death Detection kit, Fluorescein (Promega Corporation, Madison, WI, USA) according to the manufacturer's protocol. Images were captured using a fluorescent microscope.

Analysis of caspase-3 activity. Caspase 3 activity was assayed with a fluorometric kit (R\&D Systems, Inc., Minneapolis, MN, USA) following the manufacturer's protocol. Briefly, the cells were rinsed with PBS and lysed with the lysis buffer from the kit. A substrate of caspase-3 was mixed with the lysate and incubated for $60 \mathrm{~min}$. The fluorescence signal was analyzed at $405 \mathrm{~nm}$. 
Reverse transcription-quantitative polymerase chain reaction (RT-qPCR) analysis. Total cellular RNA was isolated from the cultured cells using the RNeasy Mini kit (Qiagen, Inc., Valencia, CA, USA) according to the manufacturer's protocol. RT-qPCR analysis was performed on a 96-well plate ABI Prism 7500 Sequence Detection system (Applied Biosystems; Thermo Fisher Scientific, Inc.) using a $25 \mu 1$ reaction system containing $12.5 \mu \mathrm{l} 2 \mathrm{X}$ SYBR-Green PCR Master mix (Takara Bio, Inc., Otsu, Japan), $100 \mathrm{ng}$ cDNA and $0.4 \mu \mathrm{M}$ paired primers. The cycling conditions were as follows: 40 cycles of $94^{\circ} \mathrm{C}$ for $5 \mathrm{sec}, 60^{\circ} \mathrm{C}$ for $34 \mathrm{sec}$, and $72^{\circ} \mathrm{C}$ for $30 \mathrm{sec}$. A comparative $2^{-\Delta \Delta \mathrm{Cq}}$ method (29) was used to calculate the relative expression of target genes. The sequences of $\mathrm{Bcl}-2$ primers were forward, 5'-GGATCCAGGATAACGGAGGC-3' and reverse, 5'-GATAGGCACCCAGGGTGATG-3'. The relative expression level of $\mathrm{Bcl}-2$ was normalized to the internal reference gene glyceraldehyde 3-phosphate dehydrogenase (GAPDH) with primers were forward, 5'-CCAGGTGGTCTCCTC TGA-3' and reverse, 5'-GCTGTAGCCAAATCGTTGT-3'. The level of mature miR-497 was analyzed by Stem-loop qPCR as described (30) and normalized to that of U6 with primers were forward, 5'-CTCGCTTCGGCAGCACA-3' and reverse, 5'-AACGCTTCACGAATTTGCGT-3'.

Western blot analysis. The protein was extracted with M-PER (Thermo Fisher Scientific, Inc.), quantified using a bicinchoninic acid kit (Thermo Fisher Scientific, Inc.), and $30 \mu \mathrm{g}$ samples were separated via $12 \%$ SDS-PAGE and then transferred onto polyvinylidene difluoride membrane at $100 \mathrm{~V}$ for 70 min using a mini transfer tank for electrophoresis (Bio-Rad Laboratories, Inc., Hercules, CA, USA). The membranes were blocked in $2 \%$ bovine serum albumin (Sigma-Aldrich; Merck $\mathrm{KGaA}$, Darmstadt, Germany) for $1 \mathrm{~h}$ at room temperature, and incubated with primary antibodies $(1: 1,000)$ at $4^{\circ} \mathrm{C}$ overnight. The membranes were washed in TBS with $0.1 \%$ Tween-20 (TBST) three times ( 5 min each time), and then incubated with horseradish peroxidase-conjugated goat anti-rabbit IgG secondary antibody (cat. no. ab97051; 1:3,000) for $1 \mathrm{~h}$ at room temperature. The blots were analyzed using an enhanced chemiluminescence western blotting detection system (GE Healthcare Life Sciences, Piscataway, NJ, USA). All primary antibodies were obtained from Abcam (Cambridge, UK; BCL-2; cat. no. ab32124; BAX; cat. no. ab32503; caspase-3; cat. no. ab13847; caspase-9; cat. no. ab32539; cytochrome-c; cat. no. ab133504); GAPDH (cat. no. ab9485) was used as the internal reference.

Dual luciferase reporter assay. The wild-type 3' UTR sequence of Bcl-2 containing the predicted miR-497 binding sites using bioinformatics software TargetScan (http://www.targetscan.org) and miRanda (http://cbio. mskcc.org/microrna_data/manual.html) was amplified by PCR using a human cDNA template and paired primers (sense, 5'-GAGTTGCTTTACGTGGCCTG-3'; antisense, 5'-ACATGTGTTGGGATTGCCCT-3'). The corresponding mutated 3'UTR sequence was generated by a TaKaRa MutanBEST kit (Takara Bio, Inc., Otsu, Japan) and primers (mutation-introduced-sense, 5'-CCA ATCCTG TCAGACGTTCCTGCCAAAAT-3'; antisense, 5'-GTA ACAGTTTCACTACTTATACCTTATA-3') following the manufacturer's protocol. The two sequences were separately inserted into the pMIR-Reporter vector (Ambion; Thermo Fisher Scientific, Inc.) using restriction enzymes SacI and HindIII. These constructs, in addition to pRL-TK (Promega Corporation) and miR-497/miR-NC, were co-transfected into the cultured cells using Xfect ${ }^{\mathrm{TM}}$ Polymer. The luciferase activity was assayed using the Dual-Luciferase ${ }^{\circledR}$ Reporter (DLR ${ }^{\mathrm{TM}}$ ) Assay system (Promega Corporation) following the manufacturer's protocol. All samples were assessed in triplicate, and the results were normalized relative to control Renilla activity.

Statistical analysis. All data values are presented as the mean \pm standard deviation from at least three separate determinations. Comparison of all other results was performed by one-way analysis of variance with Tukey's comparison analysis or Student's t test, and the statistical significance was analyzed using these tests. $\mathrm{P}<0.05$ was considered to indicate a statistically significant difference. GraphPad Prism 5 (GraphPad Software, Inc., La Jolla, CA, USA) was used to compare groups.

\section{Results}

Expression of miR-497 is lower and expression of Bcl-2 is elevated in MM tissues and cells. To elucidate the association between miR-497 and MM, RT-qPCR analysis was performed in $46 \mathrm{MM}$ patients/normal individuals. The expression of miR-497 was lower in patients with MM than in normal individuals (Fig. 1A). Similar experiments were performed in the RPMI-8226 and U266 cell lines, and BMSCs used as a normal control. The results were consistent with the clinical cases, when compared with the normal BMSCs; the level of miR-497 was significantly lower in MM cells (Fig. 1B). Subsequently, the expression of Bcl-2, one of the target genes of miR-497 $(20,22,23)$, detected in MM cells and BMSCs. As expected, it was found that the expression of Bcl-2, at the mRNA level and protein level, was elevated in both MM cell lines (Fig. 1C and D). These results indicated that miR-497 was downregulated but Bcl-2 was upregulated in MM cells, a negative correlation between the two.

Ectopic overexpression of miR-497 inhibits cell proliferation of MM. It was previously reported that Bcl-2 was closely associated with myeloma cell proliferation $(3,5,15)$. To investigate the effects of miR-497 on myeloma cell proliferation, miR-497 mimic (miR-497) or the matched negative control (miR-NC) were transfected into the RPMI-8266 and U266 MM cell lines to overexpress miR-497 (Fig. 2A). The cell viability and cell cycle were then analyzed through an MTT assay and flow-cytometry, respectively. The results revealed that the overexpression of miR-497 in RPMI-8266 and U266 cells led to decreased cell viability (Fig. 2B) together with altered cell cycle with the majority of cells arrested at the $\mathrm{G}_{0} / \mathrm{G}_{1}$ phase (Fig. $2 \mathrm{C}$ and $\mathrm{D}$ ).

The soft-agar colony formation assay is another common assay to examine cell proliferation $(28,31)$. In the RPMI-8266 and U266 cells, the number of colonies in the group overexpressing miR-497 was markedly decreased compared with the miR-NC and control groups (Fig. 2E). Statistical analysis 
A 守

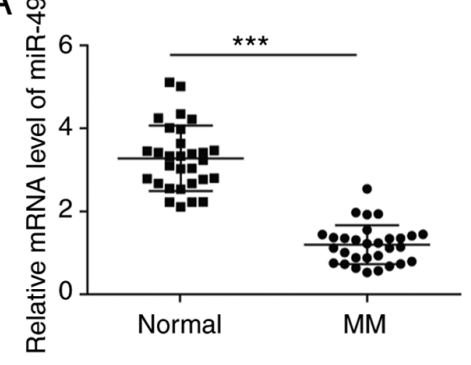

D
$\mathrm{B}$ รั

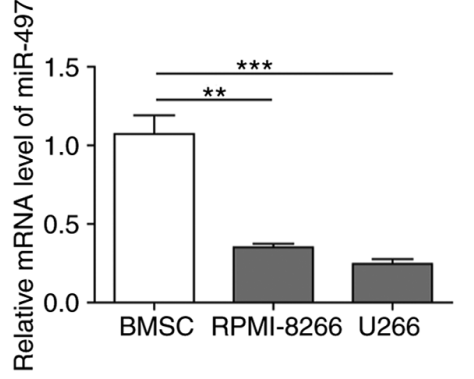

C

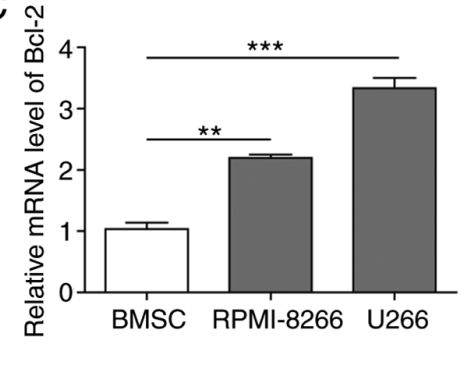

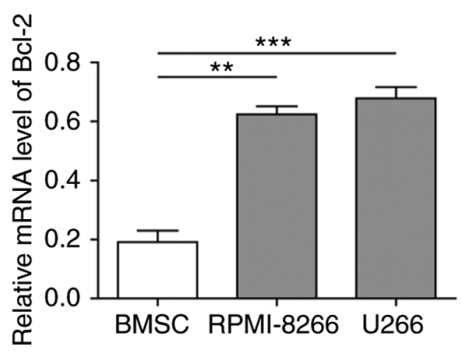

Figure 1. Expression of miR-497 is lower and that of Bcl-2 is higher in MM tissue and cells. (A) RT-qPCR analysis of levels of miR-497 in normal and MM tissues (46 cases). (B) RT-qPCR analysis of miR-497 in BMSCs (control) and RPMI-8226 and U266 MM cell lines. (C) RT-qPCR analysis of the expression level of Bcl-2 in BMSCs, RPMI-8226 and U266 cells. (D) Western blot analysis of protein levels of Bcl-2 in BMSCs, RPMI-8226 and U266 cells. GAPDH served as the internal reference. ${ }^{* *} \mathrm{P}<0.01,{ }^{* * *} \mathrm{P}<0.001$. MM, multiple myeloma; miR, microRNA; RT-qPCR, reverse transcription-quantitative polymerase chain reaction; Bcl-2, B-cell lymphoma 2.
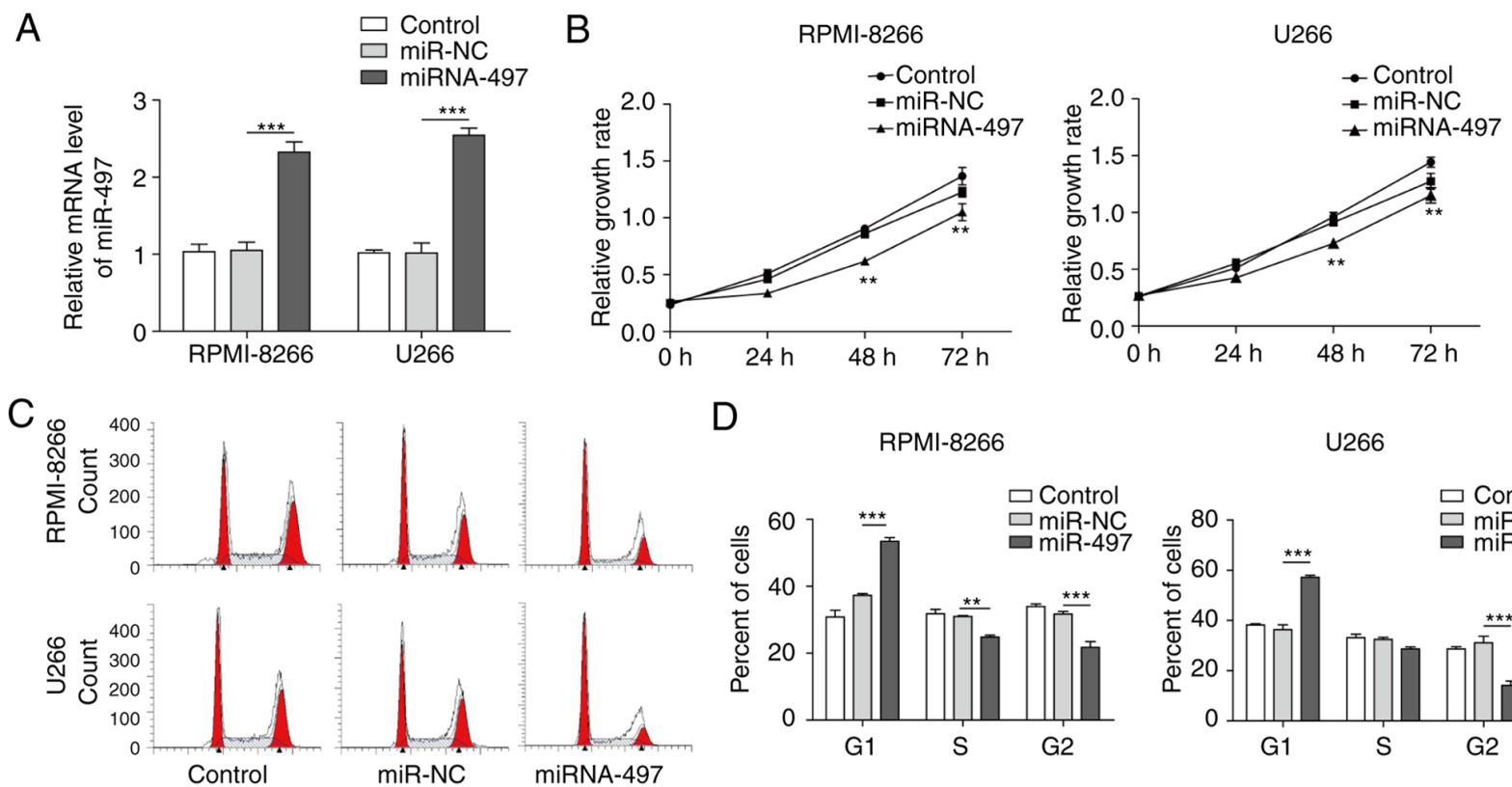

D RPMI-8266
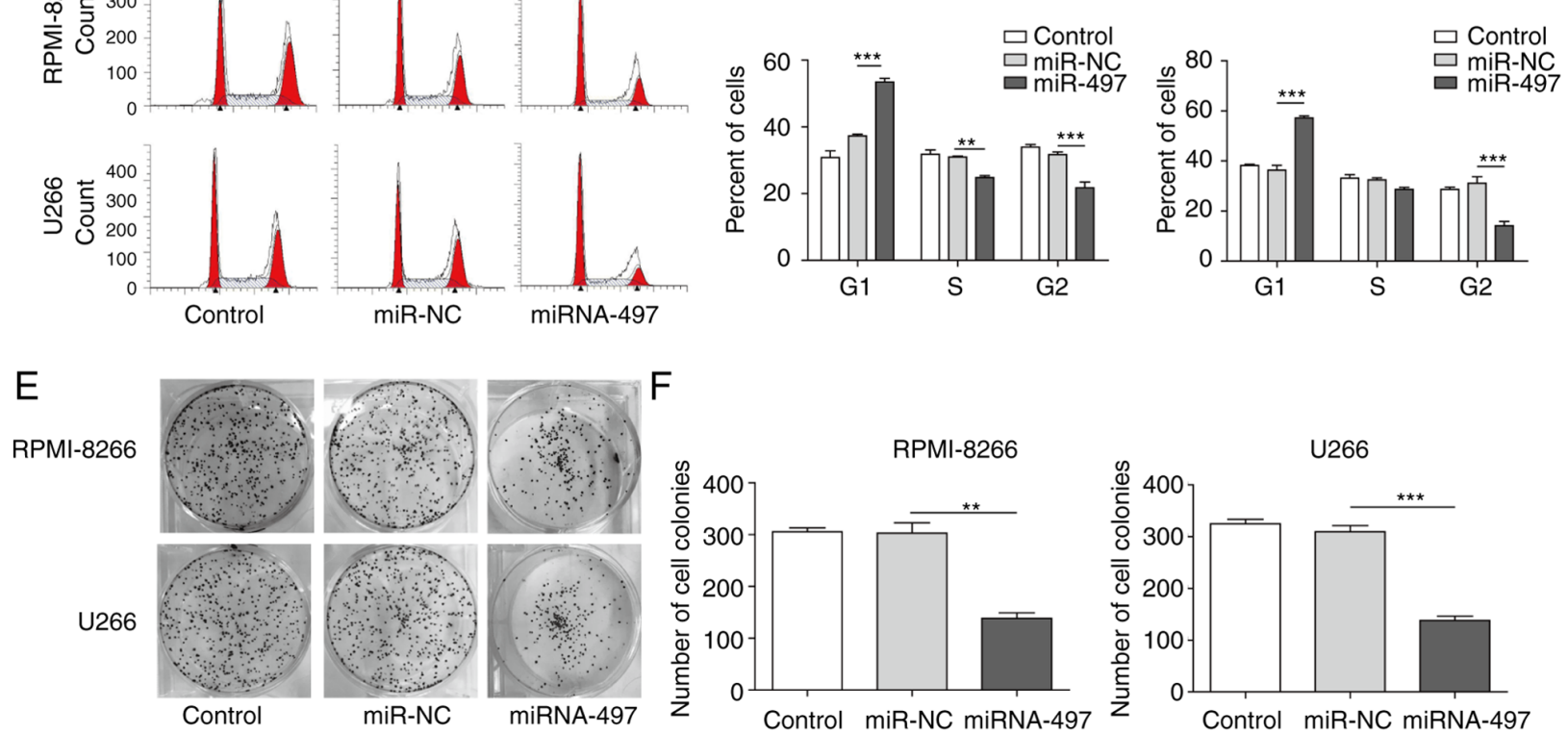

Figure 2. Ectopic overexpression of miR-497 inhibits cell proliferation of multiple myeloma cells. (A) RT-qPCR analysis of the expression of miR-497 in RPMI-8226 and U266 cells transfected with miR-497 or miR-NC, and the control group treated with nothing but equivalent transfection reagent Lipo3000. (B) Cell viability analysis using 3-(4, 5-dimethylthiazol-2-yl)-2, 5-diphenyltetrazolium bromide in cells cultured for the indicated durations. (C) Cell cycle analysis by PI staining and FACS analysis in cells transfected with miR-497 or miR-NC. (D) Percentages of cells at different cell cycle stages. (E) Soft agar colony genesis assay of cells transfected with miR-497 or miR-NC. (F) Statistical analysis indicating colony numbers. ${ }^{* *} \mathrm{P}<0.01,{ }^{* * * *} \mathrm{P}<0.001$. miR, microRNA; $\mathrm{NC}$, negative control; RT-qPCR, reverse transcription-quantitative polymerase chain reaction. 
A

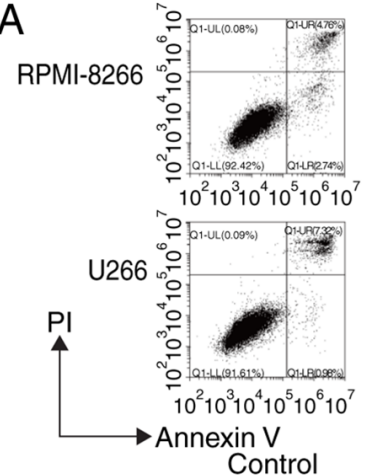

C

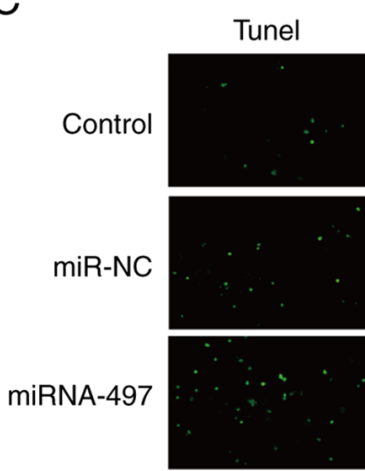

D
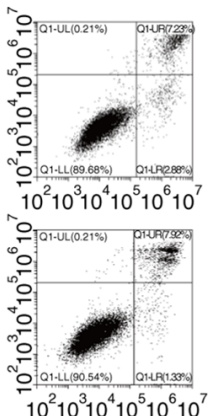

miR-NC

RPMI-8266 DAPI
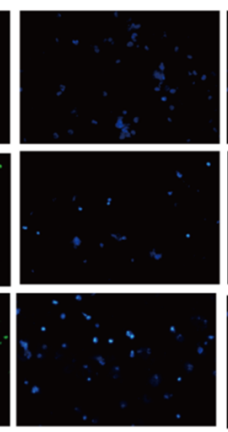

RPMI-8266

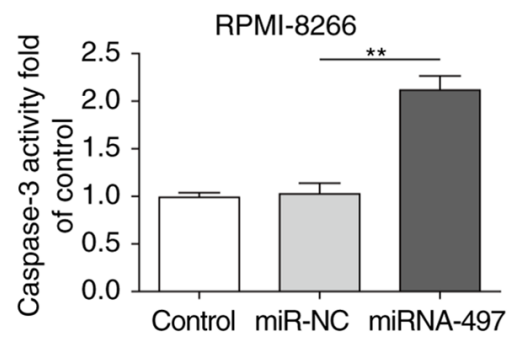

miRNA-497 Merge
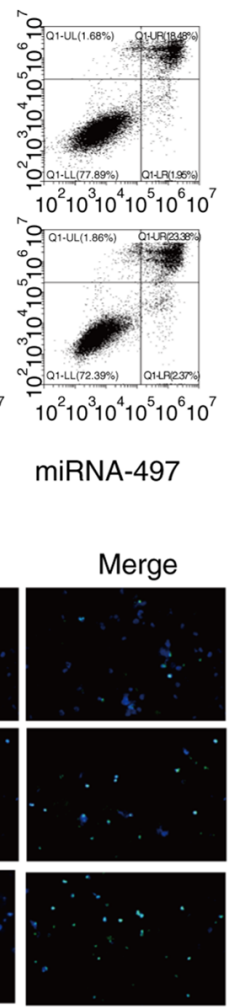

miRNA-497

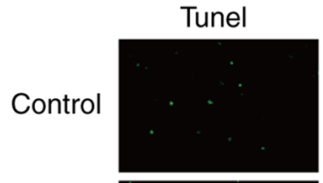

U266
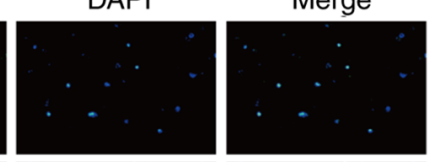

miR-NC
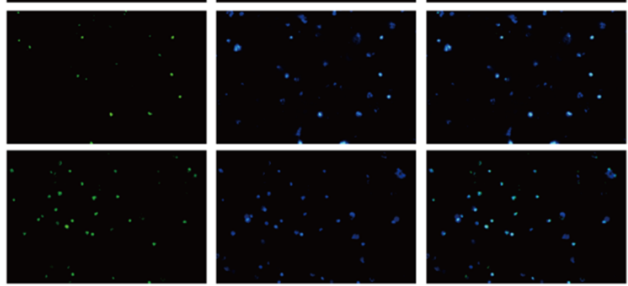

U266

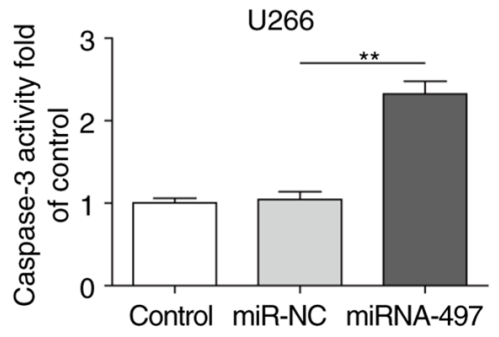

Figure 3. Overexpression of miR-497 induces multiple myeloma cell apoptosis. (A) Cell apoptosis was analyzed by Annexin V and FACS in cells transfected with miR-497 or miR-NC. (B) Statistical analysis of apoptotic cells. (C) Representative images of TUNEL fluorescent staining for analysis if apoptosis in cells transfected with miR-497 or miR-NC. Magnification, $x 100$. (D) Analysis of caspase-3 activity in cells transfected with miR-497 or miR-NC. ${ }^{* *}<0.01$. miR/miRNA, microRNA; NC, negative control; TUNEL, terminal deoxynucleotidyl transferase (TdT)-mediated dUTP nick-end-labeling.

of the colony numbers (Fig. 2F) also indicated that miR-497 disrupted clone formation of MM cells.

Taken together, the overexpression of miR-497 inhibited MM cell proliferation through cell cycle arrest, and reductions in viability and colony formation ability.

Overexpression of $\mathrm{miR}-497$ induces MM apoptosis. It was hypothesized that reduced cell viability may be caused by increased apoptosis (32-34), therefore, subsequent assays of the apoptotic patterns of cells with ectopic miR-497 were performed. Cell apoptosis was analyzed by Annexin V/FACS and TUNEL assays in RPMI-8266 and U266 cells transfected with or without miR-497. The cells with ectopic miR-497 exhibited increased apoptosis ( 2.5 fold) according to FACS results (Fig. 3A and $\mathrm{B}$ ) and TUNEL staining (Fig. 3C). To confirm these findings, the caspase- 3 activity was also detected as a common marker for apoptosis $(9,31)$. A consistent result further suggested that the overexpression of miR-497 induced apoptosis by markedly increasing the caspase- 3 activity ( 2.2 fold) (Fig. 3D). These results indicated that miR-497 promoted MM cell apoptosis.
miR-497 directly targets $\mathrm{Bcl}-2$. As a close correlation between Bcl-2 and miR-497 was described in previous reports $(20,23)$, the present study examined whether miR-497 directly targeted the expression of Bcl-2. For this, the Bcl-2 3'UTR and miR-497 sequences were analyzed by bioinformatics software, including TargetScan, miRTarBase, RNAhybrid, and a strong interaction region was found (Fig. 4A). To create an miR-497-insensitive Bcl-2 3'UTR, mutations were introduced into the binding sites (Fig. 4A), and inserted into a luciferase reporter gene plasmid, in addition to the wild-type Bcl-2 3'UTR. A dual-luciferase assay was performed to verify the interaction between miR-497 and Bcl-2. The results revealed that miR-497 significantly decreased the activity of luciferase with the wild-type Bcl-2 3'UTR, but did not affect the activity of luciferase with the mutated Bcl-2 3'UTR (Fig. 4B), suggesting that Bcl-2 serves as a direct target of $\mathrm{miR}-497$, and the interaction site is just as the prediction. To confirm this finding, the present study detected the expression levels of Bcl-2 by RT-qPCR and western blot analyses following miR-497 or miR-NC treatment. The mRNA and protein levels of Bcl-2 were 

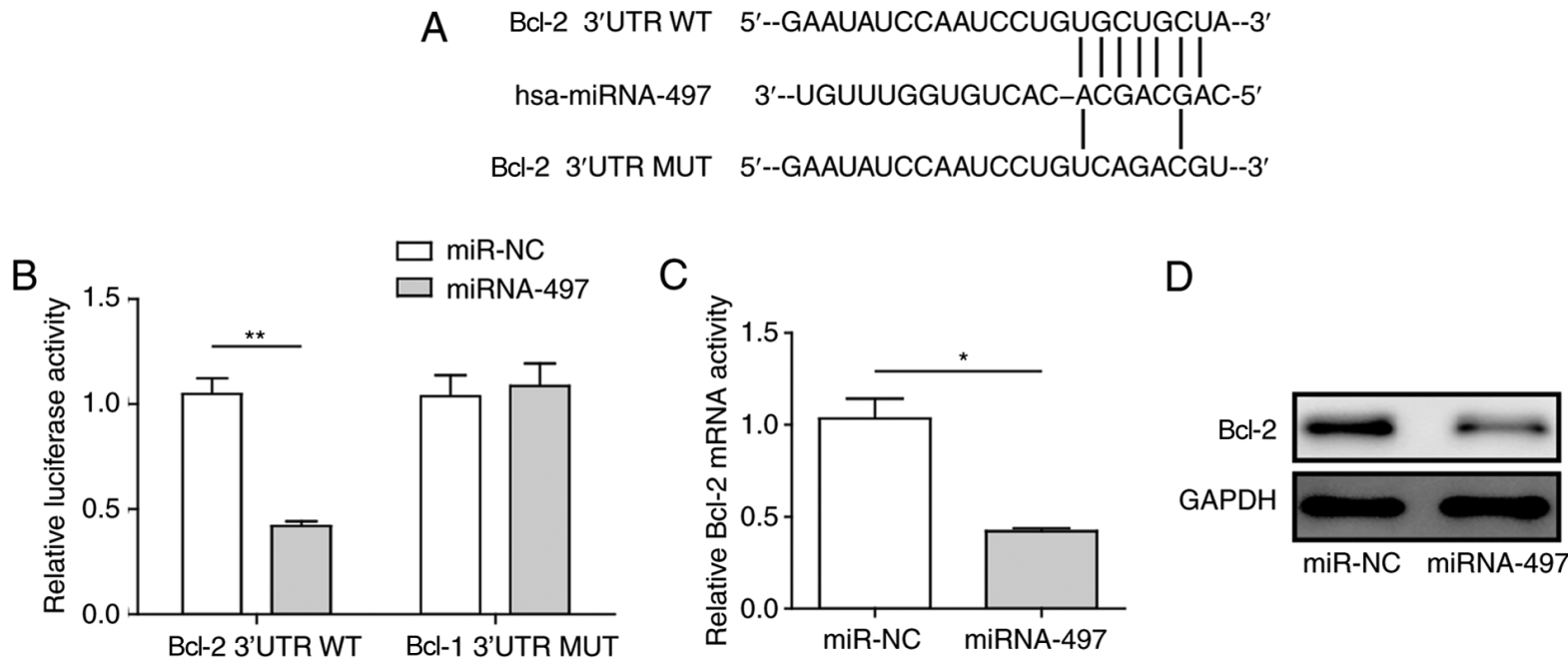

Figure 4. miR-497 directly targets Bcl-2. (A) Schematic of the miR-497 interaction with the Bcl-2 3'UTR (WT or MUT). (B) Luciferase reporter assay analysis in cells transfected with reporter plasmid inserted with wild-type or mutated Bcl-2 3'UTR sequences, in addition to miR-497 or NC as indicated. (C) Reverse transcription-quantitative polymerase chain reaction analysis of mRNA levels of Bcl-2 in cells transfected with miR-497 or miR-NC (D) Western blot analysis of protein levels of Bcl-2 in cells transfected with miR-497 or miR-NC. ${ }^{*} \mathrm{P}<0.05,{ }^{* *} \mathrm{P}<0.01$. miR/miRNA, microRNA; NC, negative control; Bcl-2, B-cell lymphoma 2; 3'UTR, 3' untranslated region; WT, wild-type; MUT, mutated.

A

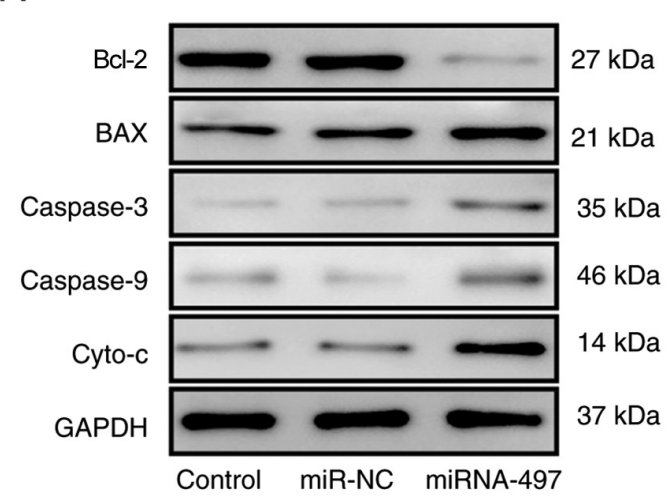

B

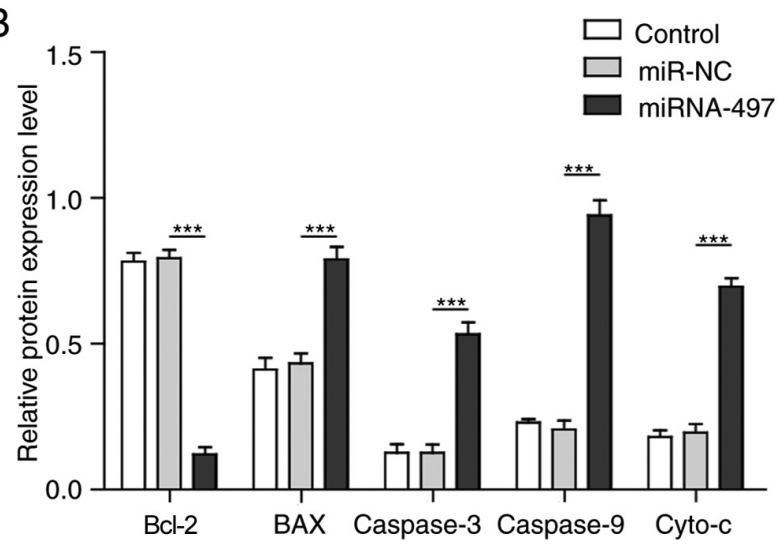

Figure 5. miR-497 regulates the expression if downstream apoptosis-related proteins by directly targeting Bcl-2. (A) Western blot analysis of Bcl-2 and its downstream apoptotic proteins in cells transfected with miR-497 or miR-NC. GAPDH served as the internal reference. (B) Statistical analysis of protein levels. The results were normalized relative to the internal reference GAPDH. ${ }^{* * *} \mathrm{P}<0.001$. miR/miRNA, microRNA; NC, negative control; Bcl-2, B-cell lymphoma 2; BAX, Bcl-2-associated X protein; cyto-c, cytochrome $c$.

markedly decreased by the overexpression of miR-497, with 3-fold inhibition (Fig. 4C and D).

miR-497 regulates the expression of downstream apoptotic-related proteins by directly targeting Bcl-2. Aside from the anti-apoptotic gene Bcl-2, other apoptotic-related proteins also closely associated with Bcl-2 and cell apoptosis. The present study detected the expression of several well-known pro-apoptotic proteins, including $\mathrm{Bcl}-2$-associated $\mathrm{X}$ protein (BAX), caspase-3, caspase-9 and cytochrome $c$ (cyto-c) by western blot analysis. All of these proteins were upregulated markedly by the overexpression of miR-497 (Fig. 5A and B).

miR-497 enhanced bortezomib chemosensitivity in MM cells. Bortezomib is a conventional clinical medication in MM therapy; however, bortezomib-resistance can also occur intrinsically or can be acquired in several patients (25). According to the findings of the present study, miR-497 inhibited proliferation and promoted apoptosis of MM cells, indicating that miR-497 may have a potential synergetic effect or sensitization to bortezomib. To confirm this hypothesis, RPMI-8226 and U266 cells, which were have been shown to be more resistant to bortezomib compared with other MM cell lines $(26,27)$, were used. Although the cell viability was decreased when treated with bortezomib treatment alone, a lower level was detected following treatment with $\geq 10$ nM bortezomib combined with miR-497 (Fig. 6A). Subsequently, $10 \mathrm{nM}$ bortezomib treatment for $24 \mathrm{~h}$ was selected for the subsequent experiments to analyze cell cycle, colony formation ability and apoptosis. The results showed that the cell cycle arrest was enhanced by the combination of miR-497 and botezomib in the RPMI-8226 and U266 cells (Fig. 6B); the proportion of cells in the $G_{0} / G_{1}$ phase increased from 54.12 to $76.25 \%$ when the cells were treated with the addition of miR-497. The colony formation ability was also decreased by the combination of miR-497 and 


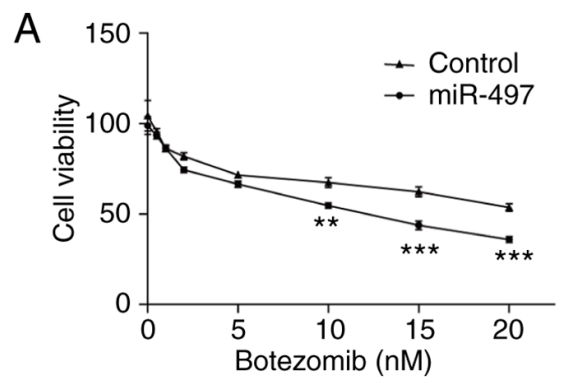

B
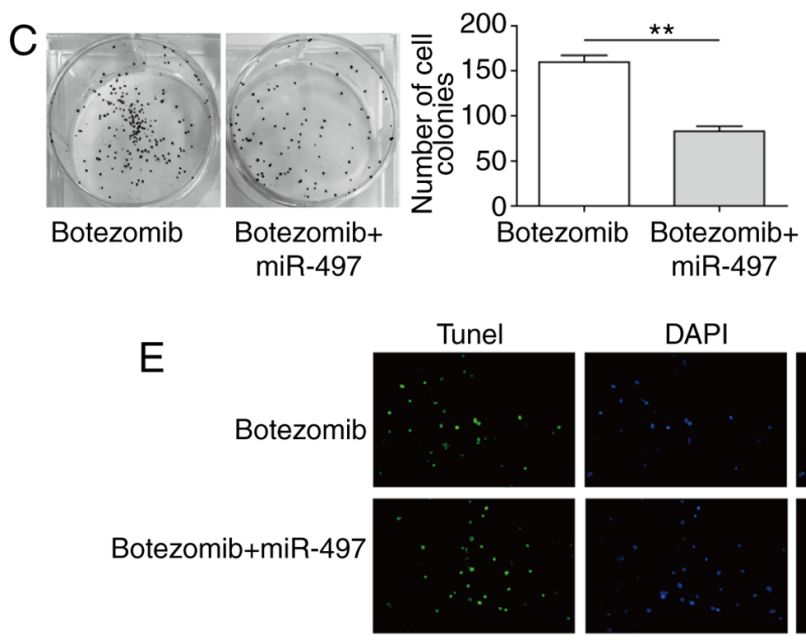
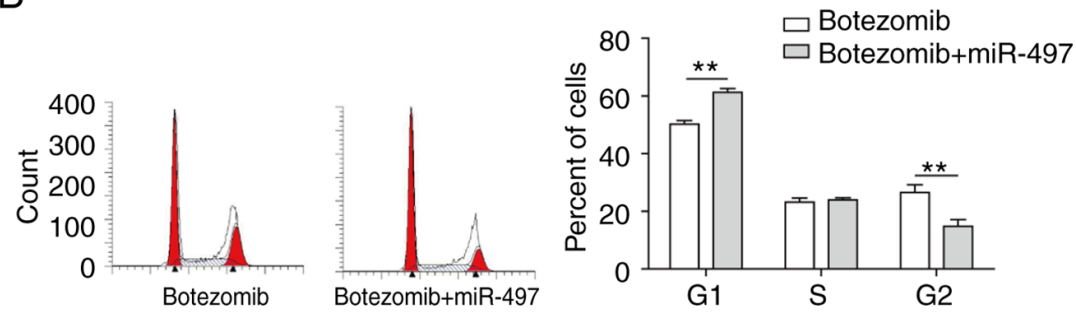
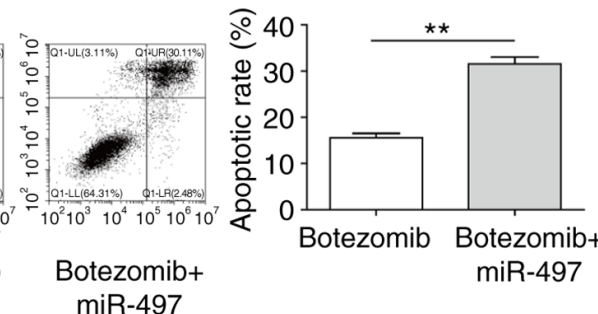
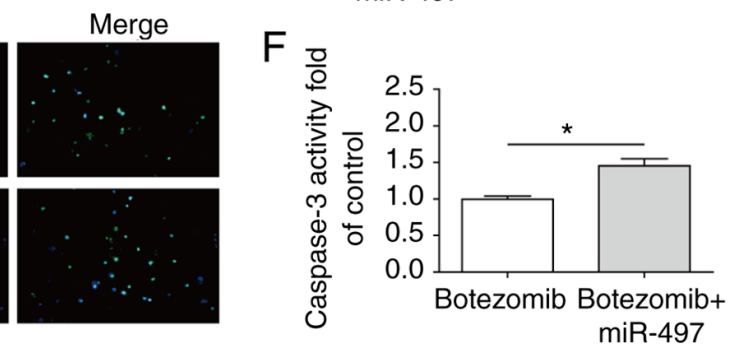

Figure 6. miR-497 enhances bortezomib chemosensitivity in MM cells. (A) Cell viability analysis by 3-(4,5-dimethylthiazol-2-yl)-2,5-diphenyltetrazolium bromide assay in MM cells treated with bortezomib and miR-497 or miR-negative control. (B) Cell cycle analysis by PI staining and flow cytometry in cells treated with bortezomib alone or in combination with miR-497. Proportions of cells at different stages were analyzed and shown in the histogram. (C) Soft agar colony formation analysis in cells treated with bortezomib and miR-497. Statistical analysis of colony numbers is shown in the histogram. (D) Analysis of apoptosis by Annexin V and FACS in cells treated as indicated. Statistical analysis of apoptotic positive cells is shown in the histogram. (E) Representative images of terminal deoxynucleotidyl transferase (TdT)-mediated dUTP nick-end-labeling fluorescent staining for apoptosis analysis in cells treated as indicated. Magnification, x100. (F) Analysis of caspase-3 activity in cells treated as indicated. ${ }^{*} \mathrm{P}<0.05,{ }^{* *} \mathrm{P}<0.01,{ }^{* * *} \mathrm{P}<0.001$. miR, microRNA; MM, multiple myeloma.

bortezomib, with $50 \%$ inhibition compared with bortezomib treatment alone (Fig. 6C). The apoptotic signature analysis by Annexin V/FACS and TUNEL assays demonstrated that miR-497 promoted bortezomib-induced MM cell apoptosis (Fig. 6D and E). As another vital hallmark of apoptosis, the activity of caspase- 3 was further detected and, as expected, miR-497 enhanced the activity of caspase- 3 when co-treated with bortezomib (Fig. 6F). Taken together, these results led to the conclusion that miR-497 enhanced the chemosensitivity of MM cells to bortezomib, and may serve as a potential therapeutic candidate in combination with bortezomib.

\section{Discussion}

MM comprises up to $10 \%$ of all hematological malignancies, ranking second to leukemia. The susceptibility to MM increases with aging. With average age increasing in China, the number of patients with MM also increases each year (1). At present, there is no completely effective cure for MM, therefore, investigating the molecular mechanism of MM is important (1). miR-497 is closely associated with multiple types of cancer, including breast cancer, ovarian cancer and glioma, by regulating proliferation, apoptosis and cell cycle (11-17). The investigation of miR-497 in MM may well provide potentially novel therapeutic ideas. The findings of the present study revealed decreased expression levels of miR-497 in MM tissues and cell lines, compared with those in control groups, supporting this potential.

By transfecting MM cells with miR-497 or miR-NC, the viability of the two MM cell lines decreased with statistical significance, as expected, in addition to the cell cycle arrest and reduced colony formation ability effects of miR-497 on MM cells. These biological processes are closely associated with carcinogenesis, and the inhibitory effect of miR-497 on these processes may lead to decreased cancer burden. The analysis of apoptotic features by TUNEL assays and Annexin V flow cytometry verified that miR-497 increased the apoptosis of MM cells. These results confirmed the hypothesis that miR-497 can have a therapeutic role against MM.

The present study then aimed to reveal the underlying mechanism of miR-497 on growth inhibition of MM cells. The first and most important step is to reveal proteins targeted by miR-497. Sequencing analyzing indicated that Bcl-2 may be a potential miR-497 target $(22,23)$, and this was validated by decreased mRNA and protein levels in MM cells transfected with miR-497. Bcl-2 is a master regulatory factor in apoptotic signaling pathways, which may regulate other apoptotic proteins levels, including BAX, caspase-3 and caspase-9. The expression of these apoptosis-related proteins was altered 
along with Bcl-2 with the overexpression of miR-497. These findings provide evidence that miR-497 directly targeted Bcl-2 and modulated apoptotic signaling in MM. Therefore, this may explain how miR-497 inhibited MM cell viability.

In consideration of the inhibitory effect of miR-497 on MM cell proliferation and viability, the present study investigated whether it can be used in MM therapy by combination with conventional medication. Bortezomib is a common drug used in MM treatment and has been shown to promote cell death of malignant plasma cells (25). The combination of miR-497 and bortezomib in MM cells confirmed a marked susceptibility increase for bortezomib treatment in the present study. In the miR-497-overexpressing groups, bortezomib administration resulted in more marked viability inhibition, more severe cell cycle arrest, increased apoptosis and more marked colony formation ability loss, compared with the miR-NC groups. These findings suggested the therapeutic effect was superior when miR-497 and bortezomib were combined together against MM cells.

The present study revealed the function of miR-497 in MM; miR-497 was downregulated in MM tissues and cells, and the overexpression of miR-497 induced MM cell apoptosis by inhibiting cell proliferation and viability. In addition, the present study revealed the underlying mechanism of miR-497 by directly targeting and inhibiting the expression of Bcl-2, upregulating the expression of pro-apoptotic genes and finally promoting apoptosis. When the combination treatment of bortezomib with miR-497 was used, enhanced chemosensitivity was readily detected in MM cells. These findings may assist in investigating the mechanisms of $\mathrm{MM}$ and provide novel potential therapeutic methods for future clinical applications.

\section{Acknowledgements}

Not applicable.

\section{Funding}

The present study was funded by the Science and Technology R\&D Fund of Shenzhen (grant no. JCYJ20170307104838077), the Shenzhen Longgang District Science and Technology Development Fund (grant no. YLWS 20150514150041453) and the National Natural Science Foundation of China (grant no. 81472275).

\section{Availability of data and materials}

All data generated or analyzed during this study are included in this published article.

\section{Authors' contributions}

YZ and WZ performed the cell culture, drugs treatment, flow cytometry and TUNEL analysis, and were major contributors in writing the manuscript. JL performed the collection and management of clinic specimens. MT and XC performed the dual luciferase reporter assays and molecular expression detections. JJ analyzed the data. FT designed the experiments, revised the paper and submitted the final versions. All authors read and approved the final manuscript.

\section{Ethics approval and consent to participate}

The use of tissue specimens was based on an informed and voluntary principle, and was reviewed and approved by the Ethics Committee of Longgang District People's Hospital of Shenzhen.

\section{Patient consent for publication}

Not applicable.

\section{Competing interests}

The authors declare that they have no competing interests.

\section{References}

1. Abdi J, Rastgoo N, Li L, Chen W and Chang H: Role of tumor suppressor p53 and micro-RNA interplay in multiple myeloma pathogenesis. J Hematol Oncol 10: 169, 2017.

2. Ahmad N, Haider S, Jagannathan S, Anaissie E and Driscoll JJ: MicroRNA theragnostics for the clinical management of multiple myeloma. Leukemia 28: 732-738, 2014.

3. Troppan K, Wenzl K, Pichler M, Pursche B, Schwarzenbacher D, Feichtinger J, Thallinger GG, Beham-Schmid C, Neumeister P and Deutsch A: miR-199a and miR-497 are associated with better overall survival due to increased chemosensitivity in diffuse large $\beta$-cell lymphoma patients. Int J Mol Sci 16: 18077-18095, 2015.

4. Rastgoo N, Abdi J, Hou J and Chang H: Role of epigenetics-microRNA axis in drug resistance of multiple myeloma. J Hematol Oncol 10: 121, 2017.

5. Sevcikova S, Kubiczkova L, Sedlarikova L, Slaby O and Hajek R: Serum miR-29a as a marker of multiple myeloma. Leuk Lymphoma 54: 189-191, 2013.

6. Fang T. Wu Q, Zhou L, Mu S and Fu Q: miR-106b-5p and miR-17-5p suppress osteogenic differentiation by targeting Smad5 and inhibit bone formation. Exp Cell Res 347: 74-82, 2016.

7. Xu D, Gao Y, Hu N, Wu L and Chen Q: miR-365 Ameliorates dexamethasone-induced suppression of osteogenesis in MC3T3-E1 cells by targeting HDAC4. Int J Mol Sci 18, 2017.

8. Tang CM, Zhang M, Huang L, Hu ZQ, Zhu JN, Xiao Z, Zhang Z, Lin QX, Zheng XL, Yang M, et al: CircRNA_000203 enhances the expression of fibrosis-associated genes by derepressing targets of miR-26b-5p, Colla2 and CTGF, in cardiac fibroblasts. Sci Rep 7: 40342, 2017.

9. Zhai Y, Tyagi SC and Tyagi N: Cross-talk of MicroRNA and hydrogen sulfide: A novel therapeutic approach for bone diseases. Biomed Pharmacother 92: 1073-1084, 2017.

10. Kang H, Chen H, Huang P, Qi J, Qian N, Deng L and Guo L: Glucocorticoids impair bone formation of bone marrow stromal stem cells by reciprocally regulating microRNA-34a-5p. Osteoporos Int 27: 1493-1505, 2016.

11. Lu Y, Li F, Xu T and Sun J: miRNA-497 Negatively regulates the growth and motility of chondrosarcoma cells by targeting Cdc25A. Oncol Res 23: 155-163, 2016.

12. Kong XJ,. Duan LJ, Qian XQ, Xu D, Liu HL, Zhu YJ and Qi J: Tumor-suppressive microRNA-497 targets IKKbeta to regulate NF-kappaB signaling pathway in human prostate cancer cells. Am J Cancer Res 5: 1795-804, 2015.

13. Lin Z, Zhao J, Wang X, Zhu X and Gong L: Overexpression of microRNA-497 suppresses cell proliferation and induces apoptosis through targeting paired box 2 in human ovarian cancer. Oncol Rep 36: 2101-2107, 2016

14. Ge L, Zheng B, Li M, Niu L and Li Z: MicroRNA-497 suppresses osteosarcoma tumor growth in vitro and in vivo. Oncol Lett 11: 2207-2212, 2016.

15. Wei C, Luo Q, Sun X, Li D, Song H, Li X, Song J, Hua K and Fang L: MicroRNA-497 induces cell apoptosis by negatively regulating Bcl-2 protein expression at the posttranscriptional level in human breast cancer. Int J Clin Exp Pathol 8: 7729-7739, 2015.

16. Shao XJ, Miao MH, Xue J, Xue J, Ji XQ and Zhu H: The down-regulation of MicroRNA-497 contributes to cell growth and cisplatin resistance through PI3K/Akt pathway in osteosarcoma. Cell Physiol Biochem 36: 2051-2062. 2015. 
17. Li W, Jin X, Deng X, Zhang G, Zhang B and Ma L: The putative tumor suppressor microRNA-497 modulates gastric cancer cell proliferation and invasion by repressing eIF4E. Biochem Biophys Res Commun 449: 235-240, 2014.

18. Yu T, Zhang X, Zhang L, Wang Y, Pan H, Xu Z and Pang X: MicroRNA-497 suppresses cell proliferation and induces apoptosis through targeting PBX3 in human multiple myeloma. Am J Cancer Res 6: 2880-2889, 2016.

19. Lionetti M, Musto P, Di Martino MT, Fabris S, Agnelli L, Todoerti K, Tuana G, Mosca L, Gallo Cantafio ME, Grieco V, et al, Biological and clinical relevance of miRNA expression signatures in primary plasma cell leukemia. Clin Cancer Res 19: 3130-3142, 2013.

20. Wu R, Tang S, Wang M, Xu X, Yao C and Wang S: MicroRNA-497 induces apoptosis and suppresses proliferation via the Bcl-2/Bax-Caspase9-Caspase3 pathway and cyclin D2 protein in HUVECs. PLoS One 11: e0167052, 2016.

21. Zhu W, Zhu D, Lu S, Wang T, Wang J, Jiang B, Shu Y and Liu P: miR-497 modulates multidrug resistance of human cancer cell lines by targeting BCL2. Med Oncol 29: 384-391, 2012.

22. Shen L, Li J, Xu L, Ma J, Li H, Xiao X, Zhao J and Fang L: miR-497 induces apoptosis of breast cancer cells by targeting Bcl-w. Exp Ther Med 3: 475-480, 2012.

23. Yadav S, Pandey A, Shukla A, Talwelkar SS, Kumar A, Pant AB and Parmar D: miR-497 and miR-302b regulate ethanol-induced neuronal cell death through BCL2 protein and cyclin D2. J Biol Chem 286: 37347-37357, 2011.

24. Zhu D, Tu M, Zeng B, Cai L, Zheng W, Su Z and Yu Z: Up-regulation of miR-497 confers resistance to temozolomide in human glioma cells by targeting $\mathrm{mTOR} / \mathrm{Bcl}-2$. Cancer Med 6: 452-462, 2017.

25. Gandolfi S, Laubach JP, Hideshima T, Chauhan D, Anderson KC and Richardson PG: The proteasome and proteasome inhibitors in multiple myeloma. Cancer Metastasis Rev 36: 561-584, 2017.

26. Huang J, Zhou Y, Thomas GS, Gu Z, Yang Y, Xu H, Tricot G and Zhan F: NEDD8 inhibition overcomes CKS1B-Induced drug resistance by upregulation of p21 in multiple myeloma. Clin Cancer Res 21: 5532-5542, 2015.
27. Campanella A, Santambrogio P, Fontana F, Frenquelli M, Cenci S, Marcatti M, Sitia R, Tonon G and Camaschella C: Iron increases the susceptibility of multiple myeloma cells to bortezomib. Haematologica 98: 971-979, 2013.

28. Florine EM, Miller RE, Porter RM, Evans CH, Kurz B and Grodzinsky AJ: Effects of dexamethasone on mesenchymal stromal cell chondrogenesis and aggrecanase activity: Comparison of agarose and Self-Assembling peptide scaffolds. Cartilage 4: 63-74, 2013.

29. Livak KJ and Schmittgen TD: Analysis of relative gene expression data using real-time quantitative PCR and the 2- $\Delta \Delta C$ T method. Methods 25: 402-408, 2001.

30. Chen C, Ridzon DA, Broomer AJ, Zhou Z, Lee DH, Nguyen JT, Barbisin M, Xu NL, Mahuvakar VR, Andersen MR, et al: Real-time quantification of microRNAs by stem-loop RT-PCR. Nucleic Acids Res 33: 179, 2005.

31. Wang YG, Han XG, Yang Y, Qiao H, Dai KR, Fan QM and Tang TT: Functional differences between AMPK alphal and alpha 2 subunits in osteogenesis, osteoblast-associated induction of osteoclastogenesis, and adipogenesis. Sci Rep 6: 32771,2016

32. Song L, Zhao J, Zhang X, Li H and Zhou Y: Icariin induces osteoblast proliferation, differentiation and mineralization through estrogen receptor-mediated ERK and JNK signal activation. Eur J Pharmacol 714: 15-22, 2013.

33. Sun J, Yan P, Chen Y, Chen Y, Yang J, Xu G, Mao H and Qiu Y: MicroRNA-26b inhibits cell proliferation and cytokine secretion in human RASF cells via the Wnt/GSK-3beta/beta-catenin pathway. Diagn Pathol 10: 72, 2015.

34. Wang L, Jiang CF, Li DM, Ge X, Shi ZM, Li CY, Liu X, Yin Y, Zhen L, Liu LZ and Jiang BH: MicroRNA-497 inhibits tumor growth and increases chemosensitivity to 5 -fluorouracil treatment by targeting KSR1. Oncotarget 7: 2660-2671, 2016. 